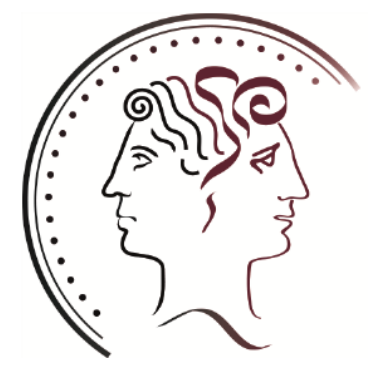

Working Papers of the Priority Programme 1859

Experience and Expectation.

Historical Foundations of Economic Behaviour

Edited by Alexander Nützenadel und Jochen Streb

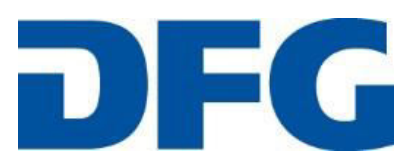

No 27 (2020, October)

Wehrheim, Lino / Jopp, Tobias A. / Spoerer, Mark

Diskurs, Narrativ, Sonderweg, Hitler, Turn

Konjunkturen geschichtswissenschaftlicher Begriffe im „Clio Viewer" 
Arbeitspapiere des Schwerpunktprogramms 1859 der Deutschen Forschungsgemeinschaft „Erfahrung und Erwartung. Historische Grundlagen ökonomischen Handelns“ /

Working Papers of the German Research Foundation's Priority Programme 1859

"Experience and Expectation. Historical Foundations of Economic Behaviour"

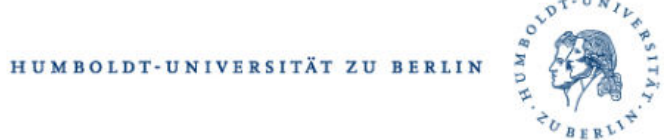

Published in co-operation with the documentation and publication service of the Humboldt University, Berlin (https://edoc.hu-berlin.de).

ISSN: 2510-053X

Redaktion: Alexander Nützenadel, Jochen Streb, Ingo Köhler

V.i.S.d.P.: Alexander Nützenadel, Jochen Streb

SPP 1859 "Erfahrung und Erwartung. Historische Grundlagen ökonomischen Handelns"

Sitz der Geschäftsführung:

Humboldt-Universität

Friedrichstr. 191-193, 10117 Berlin

Tel: 0049-30-2093-70615, Fax: 0049-30-2093-70644

Web: https://www.experience-expectation.de

Koordinatoren: Alexander Nützenadel, Jochen Streb

Assistent der Koordinatoren: Ingo Köhler

Recommended citation:

Wehrheim, Lino / Jopp, Tobias A. / Spoerer, Mark (2020): Diskurs, Narrativ, Sonderveg, Hitler, Turn. Konjunkturen geschichtswissenschaftlicher Begriffe im „Clio Viewer". Working Papers of the Priority Programme 1859 "Experience and Expectation. Historical Foundations of Economic Behaviour" No 27 (October), Berlin

(C) 2020 DFG-Schwerpunktprogramm 1859 „Erfahrung und Erwartung. Historische Grundlagen ökonomischen Handelns"

The opinions and conclusions set forth in the Working Papers of the Priority Programme 1859 Experience and Expectation. Historical Foundations of Economic Behaviour are those of the authors. Reprints and any other use for publication that goes beyond the usual quotations and references in academic research and teaching require the explicit approval of the editors and must state the authors and original source. 


\title{
Diskurs, Narrativ, Sonderweg, Hitler, Turn \\ Konjunkturen geschichtswissenschaftlicher Begriffe im „Clio Viewer“
}

\author{
Lino Wehrheim Tobias A. Jopp Mark Spoerer \\ Universität Regensburg*
}

29. Oktober 2020

\begin{abstract}
Auf Anregung einer (nicht-repräsentativen) Twitter-Umfrage unter Historikerinnen und Historikern möchten wir in diesem Beitrag ein von uns zusammengetragenes Korpus an Aufsätzen aus elf geschichtswissenschaftlichen Fachjournalen vorstellen und anhand einiger Beispiele illustrieren, wie sich der Gebrauch verschiedener prägnanter geschichtswissenschaftlicher (Leit-) Begriffe seit 1950 verändert hat. Das Ziel ist nicht, eine „digitale Begriffsgeschichte“ verschiedener Begriffe vorzulegen oder gar Rückschlüsse auf die Entwicklung des Fachs zu ziehen. Vielmehr geht es uns darum, die vorgeschlagenen und um eigene Kandidaten ergänzten Begriffe und deren Konjunkturen zu präsentieren, um so eine Grundlage für eine weitergehende Diskussion digitalhistorischer Methoden und des Nutzens eines „Clio Viewers“ zu legen. Besonderes Augenmerk richten wir auf die Erörterung der mit dem Einsatz einfacher Stichwortsuchen verbundenen methodischen Fallstricke.
\end{abstract}

JEL: A20, N01, Y80

Keywords: Historiographiegeschichte, Digital History, Begriffsgeschichte, Wortverlaufskurven, Fachzeitschriften

\footnotetext{
* Alle Autoren: Lehrstuhl für Wirtschafts- und Sozialgeschichte, Institut für Geschichte, Universität Regensburg. Kontakt: lino.wehrheim@ur.de.
} 
Das Durchsuchen umfangreicher digitaler Textbestände nach Schlagwörtern, etwa im Ngram Viewer von Google, im Digitalen Wörterbuch der Deutschen Sprache oder im Deutschen Textarchiv ${ }^{1}$, erfreut sich, trotz aller epistemologischen und technischen Grenzen, zunehmender Beliebtheit, lassen sich damit doch Begriffskonjunkturen anschaulich visualisieren und im besten Fall Rückschlüsse auf zugrunde liegende Entwicklungen ziehen. ${ }^{2}$ Das zuletzt erschienene Buch des Wirtschaftsnobelpreisträgers Robert Shiller („Narrative Wirtschaft") basiert beispielsweise im Grunde gänzlich auf entsprechenden Suchen im Google Ngram Viewer und der Zeitungsdatenbank ProQuest. ${ }^{3}$ Eine im deutschsprachigen Raum angesiedelte Möglichkeit ist das besonders aus zeithistorischer Perspektive reizvolle Tool von Zeit online, das 2019 „viral“ ging und mit dessen Hilfe die Plenarprotokolle des Deutschen Bundestags durchsucht werden können. ${ }^{4}$ So lässt sich beispielsweise veranschaulichen, dass im Bundestag [Geschichte $]^{5}$ besonders zwischen 1970 und 1990 Konjunktur hatte (siehe Abbildung 1) - eine Beobachtung, die wiederum den Ausgangspunkt einer genaueren Untersuchung der einschlägigen Plenardebatten bilden könnte. ${ }^{6}$

\section{Abbildung 1 Geschichte im Deutschen Bundestag}

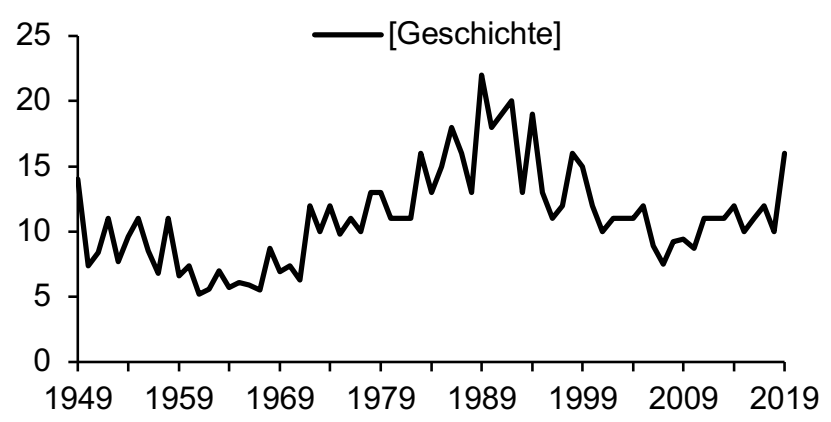

Anmerkungen: Treffer pro 100.000 Wörter. Quelle: Zeit online.

1 https://books.google.com/ngrams, https://www.dwds.de, http://www.deutschestextarchiv.de, (Zugriff am $01.10 .2020)$.

${ }^{2}$ Dieser Befund lässt sich unter anderem auch selbst als Stichwortsuche veranschaulichen, indem man mittels des Ngram Viewer nach [Ngram Viewer] durchsucht. https://books.google.com/ngrams/graph?year end=2019\&content $=$ Ngram + Viewer\&smoothing $=0 \&$ corpus $=26 \&$ year_start $=1800 \&$ direct url $=t 1 \% 3 \mathrm{~B} \% 2 \mathrm{CNgram} \% 20 \mathrm{Vie}-$ wer $\% 3 \mathrm{~B} \% 2 \mathrm{Cc} 0$ (Zugriff am 02.10.2020).

${ }^{3}$ Robert J. Shiller, Narrative Wirtschaft: Wie Geschichten die Wirtschaft beeinflussen - ein revolutionärer Erklärungsansatz (Kulmbach, 2020).

${ }^{4}$ https://www.zeit.de/politik/deutschland/2019-09/bundestag-jubilaeum-70-jahre-parlament-reden-woerter-sprache-wandel\#s=vollbeschäftigung (Zugriff am 01.10.2020).

${ }^{5}$ Auch wenn es den Lesefluss stören mag, so haben wir uns entschieden, im Folgenden konkrete Suchbegriffe aus Gründen der Nachvollziehbarkeit mit einer expliziten Notation zu versehen. Geschweifte Klammern \{\} markieren dabei einen Suchbegriff inklusive seiner Flexionen, eckige Klammern [ ] kennzeichnen einen feststehenden Suchausdruck, * markiert einen Platzhalter für die Suche mittels einer Trunkierung. Grundsätzlich blieb, soweit nicht anders angegeben, Großschreibung unberücksichtigt.

${ }^{6}$ Dafür wäre dann wiederum die Parlamentsdokumentation des Deutschen Bundestags einschlägig, die ebenfalls digital durchsuchbar ist. Siehe https://pdok.bundestag.de (Zugriff am 16.10.2020). 
Diese Möglichkeiten werden mittlerweile auch von Historikerinnen und Historikern genutzt, wie etwa von Philipp Sarasin in einem Aufsatz über Michel Foucault. ${ }^{7}$ In unserem Korpus finden sich nur - oder immerhin - elf Aufsätze, die von dem 2010 eingeführten Ngram Viewer ${ }^{8}$ in der einen oder anderen Weise Gebrauch machen oder ihn zumindest erwähnen, wovon die ersten beiden im Jahr 2011 in den Vierteljahrsheften für Zeitgeschichte erschienen sind. ${ }^{9}$ Auch und gerade für die Frage nach wissenschaftshistorischen Trends ergeben sich mit der zunehmenden digitalen Verfügbarkeit ganzer Zeitschriftenbestände und methodischer Ansätze wie dem Text Mining, also der computergestützten Textanalyse, grundsätzlich eine Vielzahl neuer Möglichkeiten. Dies hat, wie die wachsende Literatur in diesem Bereich deutlich zeigt, zu einem enormen Interesse von Wissenschaftlerinnen und Wissenschaftlern aller Couleur am jeweils eigenen Fach geführt, wobei häufig, aber nicht immer, komplexere Ansätze wie beispielsweise Topic Modelling zum Einsatz kommen. ${ }^{10}$ Doch auch wenn die Zahl digital durchsuchbarer Textbestände und die Möglichkeiten für deren computergestützte Auswertung zunehmen, stellt sich doch häufig das Problem, dass gerade mit Blick auf das eigene Forschungsinteresse

\footnotetext{
${ }^{7}$ Philipp Sarasin, „Sozialgeschichte vs. Foucault im Google Books Ngram Viewer“, in Pascal Maeder, Barbara Lüthi und Thomas Mergel, Hrsg., Wozu noch Sozialgeschichte? (Göttingen, 2012), S. 151-174.

${ }^{8}$ J.-B. Michel, Y. K. Shen, A. P. Aiden, A. Veres, M. K. Gray, J. P. Pickett, D. Hoiberg, D. Clancy, P. Norvig, J. Orwant, S. Pinker, M. A. Nowak und E. L. Aiden, „Quantitative Analysis of Culture Using Millions of Digitized Books“, Science, 331 (2011), S. 176-182.

9 Frank Bösch, „Transnationale Revolutionen. Die Bundesrepublik und die Systemwechsel im Iran und in Nicaragua“, Archiv für Sozialgeschichte, 59 (2019), S. 271-286; Alexander Engel, „,Ist nämlich der ganze Spekulationsverkehr erst einmal in einen krankhaft erregten Zustand hineingerathen...' Pathologien der Börse im späten 19. Jahrhundert“, Jahrbuch für Wirtschaftsgeschichte, 57 (2016), S. 333-365; Rüdiger Graf, „Verhaltenssteuerung jenseits von Markt und Moral“, Vierteljahrshefte für Zeitgeschichte, 66 (2018), S. 435-462; Rüdiger Graf und Kiran Klaus Patel, „Zeitgeschichte in der Welt der Sozialwissenschaften. Legitimität und Originalität einer Disziplin“, Vierteljahrshefte für Zeitgeschichte, 59 (2011), S. 479-508; Samuel Moyn, „Die neue Historiographie der Menschenrechte“, Geschichte und Gesellschaft, 38 (2012), S. 545-572; Kiran Klaus Patel, „Zeitgeschichte im digitalen Zeitalter: Neue und alte Herausforderungen“, Vierteljahrshefte für Zeitgeschichte (2011), S. 331-351; Thomas Pittrof, „,Dieser Versuch ist leider insofern ALS GESCHEITERT ZU BETRACHTEN`: Das Ende von ,Hochland“ (1971) und ,NEUEM HOCHLAND‘ (1974) - Kennmarke(n) in der Geschichte des Deutschen Nachkriegskatholizismus?", Historisches Jahrbuch, 136 (2016), S. 7-36; Kim Christian Priemel, „Spekulation als Gegenstand historischer Forschung“, Jahrbuch für Wirtschaftsgeschichte (2013), S. 926; Matthias Schmelzer, „,Expandiere oder Stirb“. Wachstumsziele, die OECD und die Steigerungslogik wirtschaftlicher Expansion“, Geschichte und Gesellschaft, 41 (2015), S. 355-393; Daniel Speich Chassé, „Die ,Dritte Welt‘ als Theorieeffekt. Ökonomisches Wissen und globale Differenz“, Geschichte und Gesellschaft, 41 (2015), S. 580-612; Oliver Zimmer, „Die Ungeduld mit der Zeit. Britische und deutsche Bahnpassagiere im Eisenbahnzeitalter", Historische Zeitschrift, 308 (2019), S. 46-80.

${ }^{10}$ Um nur einige Beispiele aus dem Bereich der Wirtschafts- und Sozialgeschichte, den wir am besten überblicken, zu nennen: Michael Buchner, Tobias A. Jopp, Mark Spoerer und Lino Wehrheim, ,Zur Konjunktur des Zählens oder wie man Quantifizierung quantifiziert. Eine empirische Analyse der Anwendung quantitativer Methoden in der deutschen Geschichtswissenschaft", Historische Zeitschrift, 310 (2020), S. 580-621; Martina Cioni, Giovanni Federico und Michelangelo Vasta, „The long-term evolution of economic history: evidence from the top five field journals (1927-2017)“, Cliometrica, 14 (2020), S. 1-39; Claude Diebolt und Michael Haupert, „A cliometric counterfactual: what if there had been neither Fogel nor North?", Cliometrica, 12 (2018), S. 407-434; Gregori Galofré-Vilà, „The Past's Long Shadow: A Systematic Review and Network Analysis of Economic History“, Research in Economic History, 36 (2020), S. 109-124; Steven Ruggles und Diana L Magnuson, „The History of Quantification in History: The JIH as a Case Study“, The Journal of Interdisciplinary History, 50 (2019), S. 363381; Lino Wehrheim, „Economic History Goes Digital: Topic Modeling the Journal of Economic History“, Cliometrica, 13 (2019), S. 83-125.
} 
(noch) keine passende Textsammlung zur Verfügung steht. ${ }^{11}$ So fand sich lange Zeit kein umfassenderes Korpus, das beispielsweise die deutschsprachige Geschichtswissenschaft einigermaßen repräsentativ abbildete und damit die Analyse von Trends in unserem Fach einem digitalen Zugang öffnete. ${ }^{12}$ Diesen Mangel haben wir mit der Erhebung eines Korpus aus deutschsprachigen Fachzeitschriften für den Zeitraum zwischen 1951 und 2016 zu lindern versucht. Auf Basis jenes Korpus konnten wir der Frage nachgehen, wie stark der Einsatz quantitativer Methoden in der deutschen Geschichtswissenschaft verbreitet war bzw. ist. ${ }^{13}$ Wie wir unter anderem mittels eines Wörterbuchansatzes, also der Abfrage nicht nur eines, sondern gleich einer ganzen Reihe von Begriffen, zeigen konnten, ist der gemeinhin auf die 1960er und 1970er Jahren datierte Trend hin zur Messung und Quantifizierung ${ }^{14}$ in der deutschen Geschichtswissenschaft nur sehr partiell nachzuweisen. Das ursprünglich zehn Zeitschriften umfassende Korpus haben wir für diesen Beitrag und ein Folgeprojekt erweitert und etwas umstrukturiert, sodass es nun elf Zeitschriften für die Zeit von 1950 bis 2019 umfasst.

Während es im Folgeprojekt darum gehen soll, das Korpus mittels eines Ansatzes zur computergestützten Inhaltsanalyse, sogenannten Topic Models, auszuwerten, setzen wir im vorliegenden Beitrag quasi einen Schritt früher an, indem wir uns auf die Erhebung und Visualisierung von Wortverlaufskurven ähnlich dem Ngram Viewer konzentrieren. Unsere Einschätzung, dass bereits diese einfachen Stichwortsuchen interessante Einblicke in die Geschichte unseres Fachs erlauben, ${ }^{15}$ regte uns zur Durchführung einer - wohlgemerkt, nicht-repräsentativen - TwitterUmfrage unter Historikerinnen und Historikern an, die einer der Autoren im Herbst 2020 durchführte. Dabei wurde um die Nennung geeigneter Kandidaten für „geschichtswissenschaftliche Modewörter" gebeten (siehe Abbildung 2). ${ }^{16}$

\footnotetext{
${ }^{11}$ So lässt sich etwa die umfassende Datenbank JSTOR zwar nach Stichworten durchsuchen, was die Literaturrecherche enorm vereinfacht; die Häufigkeit der gefundenen Begriffe lässt sich jedoch nicht ohne weiteres à la Ngram-Viewer visualisieren.

${ }^{12}$ Mit dem Deutschen Textarchiv existiert zwar ein umfangreiches Korpus historischer deutscher Textquellen. Doch geschichtswissenschaftliche Texte, vor allem serielle Quellen wie Fachzeitschriften und Buchreihen, sind unserem Wissens nach noch nicht in vergleichbarer Weise systematisch zusammengetragen worden, zumindest nicht für das 20. Jahrhundert.

${ }^{13}$ Buchner, Jopp, Spoerer und Wehrheim, „Zur Konjunktur des Zählens - oder wie man Quantifizierung quantifiziert. Eine empirische Analyse der Anwendung quantitativer Methoden in der deutschen Geschichtswissenschaft".

${ }^{14}$ Der britische Historiker Geoffrey Barraclough meinte 1971 gar einer „quantitativen Revolution“ beizuwohnen. Geoffrey Barraclough, Main Trends in History (London, New York, 1991), S. 84.

${ }^{15}$ Es stellt sich die grundsätzliche Frage, ob es aus Anwendungssicht tatsächlich immer die technisch neueste bzw. anspruchsvollste Lösung braucht, um inhaltlichen Erkenntnisfortschritt zu generieren.

${ }^{16}$ Der Aufruf ist zu finden unter https:/twitter.com/lino wehrheim/status/1309856835890012160 (Zugriff am 01.10.2020). Wie die entsprechende Tweet-Statistik mitteilt, wurde der Aufruf Stand Mitte Oktober von 2.458 Personen wahrgenommen, 278 Personen haben auf verschiedene Arten und Weisen, beispielsweise durch eine Antwort oder einen „Retweet“, damit interagiert.
} 


\section{Abbildung 2 Twitter-Umfrage}

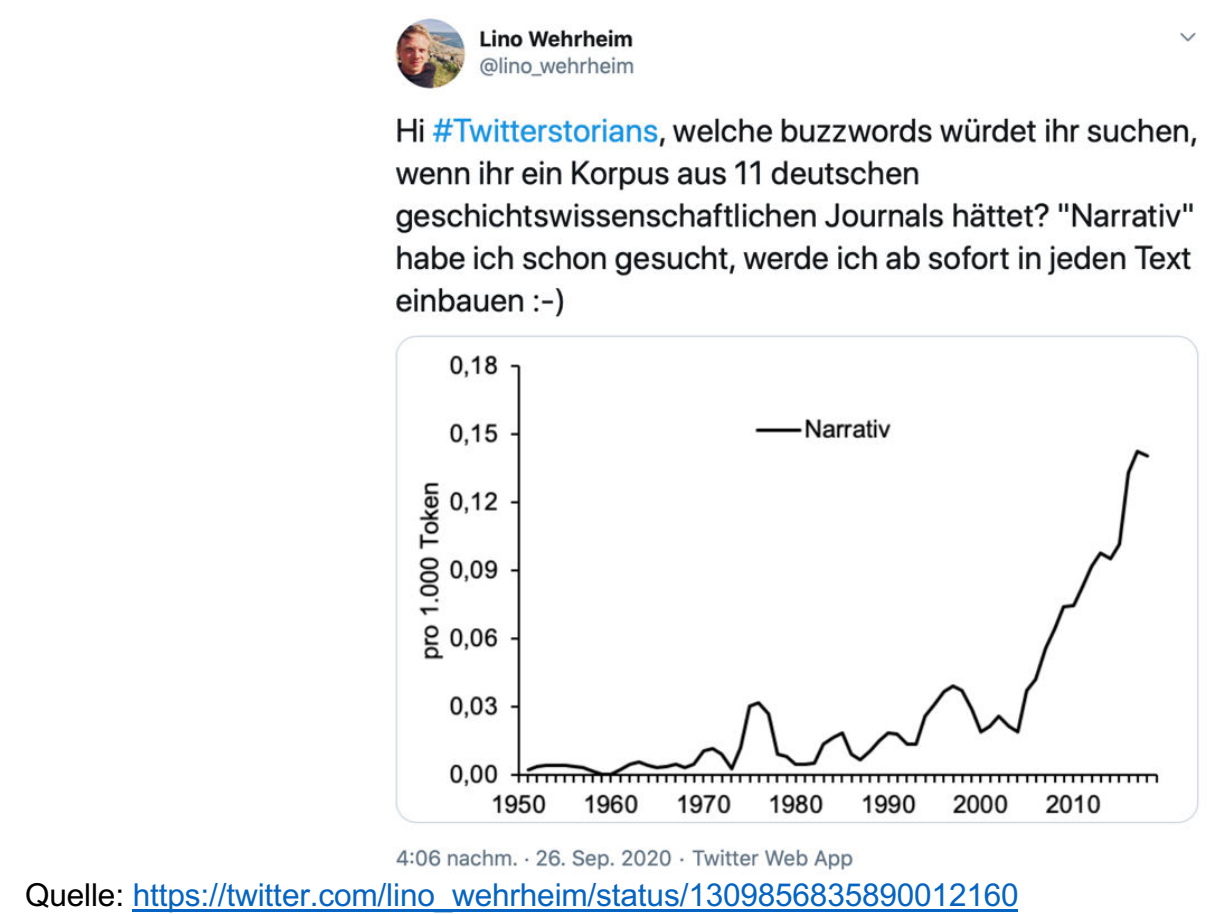

Um sowohl den zahlreichen Vorschlägen der Kolleginnen und Kollegen als auch unseren eigenen Kandidaten Raum zu geben, entschlossen wir uns, die entsprechenden Begriffskonjunkturen im vorliegenden Beitrag vorzustellen, ohne dabei den Anspruch zu erheben, eine kohärente Geschichte der deutschen Geschichtswissenschaft auf Basis von fortgeschrittenen digitalen Methoden zu formulieren. Es soll weniger um eine Einordnung der dargestellten Begriffskonjunkturen oder gar ihre Interpretation im Hinblick auf die Entwicklung des Fachs gehen, als vielmehr darum, die gesammelten Vorschläge zu präsentieren und deren Auftreten in unserem Textkorpus zu visualisieren, also im Grunde die Rohfassung eines „Clio Viewers“ vorzustellen. ${ }^{17}$ Wir gehen damit gewissermaßen einen ähnlichen und doch anderen Weg im Vergleich zu dem, was Silke Schwandt am Beispiel des lateinischen Wortes „virtus“ vorgestellt hat. ${ }^{18}$ Während Schwandt eine Vielzahl verschiedener quantitativer Auswertungen eines einzelnen Wortes anstellt, beschränken wir uns auf eine einzelne Kenngröße, die Erstellung von Wortverlaufskurven, wenden diese dafür aber auf eine Vielzahl von Begriffen an, was zwangsläufig zu einer weniger tiefgehenden, dafür breiteren Darstellung führt. Die dabei präsentierten Begriffe erheben in keiner Weise den Anspruch, das Fach in seiner Vielfalt zu repräsentieren, dazu

\footnotetext{
${ }^{17}$ Natürlich wäre es theoretisch möglich, auf Basis des Korpus eine eigene Suchmaschine analog etwa zum Tool der Zeit zu entwickeln. Hier ergibt sich jedoch, neben anderen Problemstellungen, ein nicht unerheblicher technischer Aufwand. Zudem planen wir noch einige weitere Forschungsprojekte, weshalb wir das Korpus zunächst nicht veröffentlichen werden. Für Anregungen sind wir jedoch natürlich jederzeit offen.

${ }^{18}$ Silke Schwandt, „Digitale Methoden für die Historische Semantik. Auf den Spuren von Begriffen in digitalen Korpora“, Geschichte und Gesellschaft, 44 (2018), S. 107-134. Diese Gegenüberstellung soll illustrieren, auf wie vielen Betrachtungsebenen der Einsatz digitaler Methoden gewinnbringend eingesetzt werden kann.
} 
würde allein der Platz nicht ausreichen. Vermutlich werden alle Leserinnen und Leser ad hoc Begriffe aus ihrem Forschungsbereich nennen können, die hier nicht vertreten sind. Deshalb ist unser Beitrag weniger als eigenständige bzw. umfassende Analyse denn als Diskussionsanregung zu verstehen.

\section{II.}

Grundsätzlich führt das hier vorgestellte Vorhaben, wie auch der zitierte Beitrag von Silke Schwandt beschreibt, in den Bereich der Begriffsgeschichte und der historischen Semantik. ${ }^{19}$ Zwar wird uns im Folgenden vor allem Reinhart Koselleck als Doyen der Begriffsgeschichte immer wieder begegnen. Jedoch liegen im konkreten Fall keine historischen Quellen im klassischen Sinne vor, sondern gewissermaßen Quellen auf der Metaebene, da es uns um die Praxis des geschichtswissenschaftlichen Arbeitens geht. Unseres Erachtens kann der digitale Zugang über einfache Wortfrequenzen oder auch komplexere Verfahren jedenfalls auch ein nützliches Hilfsmittel für begriffsgeschichtliche Arbeiten im klassischen Sinne sein. Dieser methodischen Vorbemerkung müssen vor der Präsentation der Ergebnisse der Stichwortsuchen einige weitere technische Vorbemerkungen folgen, da dieser Ansatz natürlich mit einigen Limitationen verbunden ist. Tatsächlich lassen sich die meisten Kritikpunkte, die für den Google Ngram Viewer formuliert wurden, auch auf den vorliegenden Fall übertragen. ${ }^{20}$

Transparenz, Repräsentativität. Erstens gilt es mit Blick auf unser Korpus festzuhalten, dass die elf Zeitschriften natürlich nur ein näherungsweises Abbild der deutschsprachigen Geschichtswissenschaft in Hinsicht auf Themenvielfalt und Sprachgebrauch liefern. Für Details

19 Bernhard Jussen, „Historische Semantik aus der Sicht der Geschichtswissenschaft“, Jahrbuch für Germanistische Sprachgeschichte (2011), S. 51-61; Reinhart Koselleck, Begriffsgeschichten (Frankfurt am Main, 2010); Ernst Müller und Falko Schmieder, Begriffsgeschichte und historische Semantik (Berlin, 2016); Otto Brunner, Werner Conze und Reinhart Koselleck, Hrsg., Geschichtliche Grundbegriffe. Historisches Lexikon zur politisch-sozialen Sprache in Deutschland (Stuttgart, 1972). Im Kontext des Digitalen ebenfalls aufschlussreich sind die Aufsätze von Alexander Friedrich und Chris Biemann, „Digitale Begriffsgeschichte? Methodologische Überlegungen und exemplarische Versuche am Beispiel moderner Netzsemantik“, E-Journal Forum interdisziplinäre Begriffsgeschichte, 5 (2016), S. 78-96, Daniel Burckhardt, Alexander Geyken, Achim Saupe und Thomas Wernecke, „Distant Reading in der Zeitgeschichte: Möglichkeiten und Grenzen einer computergestützten Historischen Semantik am Beispiel der DDR-Presse“, Zeithistorische Forschungen, 16 (2019), S. $177-196$ und Schwandt, „Digitale Methoden für die Historische Semantik. Auf den Spuren von Begriffen in digitalen Korpora“. 20 Siehe etwa Friedrich und Biemann, „Digitale Begriffsgeschichte? Methodologische Überlegungen und exemplarische Versuche am Beispiel moderner Netzsemantik“; Alexander Koplenig, „The Impact of Lacking Metadata for the Measurement of Cultural and Linguistic Change Using the Google Ngram Data Sets Reconstructing the Composition of the German Corpus in Times of WWII", Digital Scholarship in the Humanities, 32 (2015), S. 169-188; Eitan Adam Pechenick, Christopher M. Danforth und Peter Sheridan Dodds, „Characterizing the Google Books Corpus: Strong Limits to Inferences of Socio-Cultural and Linguistic Evolution“, PloS one, 10 (2015), S. na; C. Annemieke Romein, Max Kemman, Julie M. Birkholz, James Baker, Michel De Gruitjer, Albert Meroño-Peñuela, Thorsten Ries, Ruben Ros und Stefania Scaglioa, ,State of the Field: Digital History“، History, 105 (April 2020), S. 291-312. 
zur Datenerhebung sei auf unseren Ursprungsbeitrag verwiesen. ${ }^{21}$ Hier sei nur angemerkt, dass die Zeitschriften aufgrund ihrer unterschiedlichen Gründungsdaten zeitversetzt in das Korpus eintreten, was es bei der Interpretation der Ergebnisse natürlich zu bedenken gilt. Sollte etwa die Trefferhäufigkeit eines Stichwortes wie „sozial“ in den 1970er Jahren nach oben schnellen, dürfte das höchstwahrscheinlich auf die Gründung von Geschichte und Gesellschaft als Organ der aufstrebenden Historischen Sozialwissenschaft zurückzuführen sein. So gesehen spiegeln die strukturellen Veränderungen des Korpus eben auch institutionell-disziplinäre Veränderungen des Fachs wider. Ähnliches gilt für das Jahrbuch für Wirtschaftsgeschichte, das ursprünglich in der DDR erschien und das wir deswegen erst ab 1991 berücksichtigt haben. Natürlich sind diese Brüche im Korpus nicht unproblematisch, wohl aber unvermeidbar, soll ein längerer Zeitraum auf breiter Basis untersucht werden. Außerdem ist zu konzedieren, dass gerade in Subdisziplinen wie der Wirtschaftsgeschichte oder der Globalgeschichte in den letzten beiden Jahrzehnten Ergebnisse der deutschsprachigen Forschung zunehmend in englischsprachigen Zeitschriften publiziert worden sind. Dies beeinflusst die Repräsentativität, die wir hier jedoch ohnehin nicht anstreben. In der Summe beläuft sich unser Korpus auf etwas mehr als 9.000 Artikel, was einer Textmenge von gut 94 Millionen Wörtern entspricht (siehe die Übersicht über das Korpus in Tabelle 1).

Tabelle 1 Korpus

\begin{tabular}{lrrr}
\hline Zeitschrift & Erhoben ab & \# Artikel & \multicolumn{1}{c}{ Token } \\
\hline Archiv für Kulturgeschichte (AfK) & 1951 & 1.065 & 9.845 .193 \\
Archiv für Sozialgeschichte (AfS) & 1961 & 670 & 10.287 .724 \\
Geschichte und Gesellschaft (GG) & 1975 & 936 & 8.693 .514 \\
Historisches Jahrbuch (HJ) & 1951 & 760 & 7.656 .224 \\
Historische Zeitschrift (HZ) & 1950 & 1.226 & 13.583 .803 \\
Jahrbuch für Wirtschaftsgeschichte (JWG) ${ }^{1)}$ & 1991 & 378 & 3.438 .883 \\
Saeculum (Saec) & 1950 & 1.136 & 10.940 .251 \\
Vierteljahrshefte für Zeitgeschichte (VfZ) & 1953 & 1.077 & 12.614 .353 \\
Vierteljahrsschrift für Sozial- und Wirtschaftsgeschichte & 1951 & 726 & 6.756 .526 \\
(VSWG) & 1974 & 515 & 5.866 .750 \\
Zeitschrift für Historische Forschung (ZHF) & 1956 & 580 & 4.526 .209 \\
Zeitschrift für Unternehmensgeschichte (ZUG) & & 9.069 & 94.209 .430 \\
\hline Total & &
\end{tabular}

Anmerkungen: ${ }^{1)}$ Alle Artikel aus dem Jahr 2019 sind auf Englisch erschienen und wurden daher nicht berücksichtigt. Quelle: eigene Erhebung.

\footnotetext{
${ }^{21}$ Vgl. Buchner, Jopp, Spoerer und Wehrheim, „Zur Konjunktur des Zählens - oder wie man Quantifizierung quantifiziert. Eine empirische Analyse der Anwendung quantitativer Methoden in der deutschen Geschichtswissenschaft“".
} 
Messung. Zweitens ist hinsichtlich des Vorgehens der Stichwortsuche zu erwähnen, dass die absolute Zahl der Suchtreffer jeweils ins Verhältnis zur gesamten Textmenge des betrachteten Zeitraums gesetzt wird, um Veränderungen des Textumfangs - etwa aufgrund des Hinzutretens einer neuen Zeitschrift oder einer Zunahme der Artikellänge - zu berücksichtigen. Diese Relativierung bzw. Standardisierung - man spricht nun von Frequenzen - ist zwingend geboten, da ein steigender Trend in der absoluten Worthäufigkeit lediglich auf einen steigenden Textumfang zurückgehen könnte, wodurch er im Grunde keine Aussagekraft besäße. Daher ist im Folgenden die Häufigkeit eines Stichworts pro 100.000 Wörter angegeben. Aufgrund der teilweise sehr volatilen Verläufe und um die Lesbarkeit zu verbessern sind die Zeitreihen zudem mittels eines zentrierten gleitenden Drei-Jahresdurchschnitts geglättet. Dabei gilt es zu beachten, dass gerade Begriffe mit relativ niedrigen Trefferzahlen sehr anfällig für statistische Ausreißer sind. ${ }^{22}$

Fallstricke. Drittens ist eine einfache Stichwortsuche mit einigen Fallstricken verbunden, die wir anhand des folgenden Beispiels veranschaulichen möchten. ${ }^{23}$ Sucht man nach dem Ausdruck [turn], wie beispielsweise in cultural turn, so ergeben sich insgesamt 1.155 Treffer in 482 Artikeln. Dabei liegt mit 31 Treffern ein Aufsatz von Martin L. Müller aus dem Jahr 1993 auf dem dritten Platz. ${ }^{24}$ In diesem Aufsatz geht es jedoch nicht um den cultural oder irgendeinen anderen geschichtswissenschaftlichen turn, sondern um „Körperkultur in Frankfurt am Main während des Kaiserreichs und der Weimarer Republik“; die 31 Treffer resultieren aus verschiedenen Ausdrücken wie etwa „Turn- und Sportverein“. Diese offensichtlich falsch-positiven Ergebnisse (false positives) resultieren aus der Tatsache, dass manche Worte mehrere, zum Teil sehr unterschiedliche Bedeutungen besitzen; in den Sprachwissenschaften spricht man von Polysemie bzw. Homonymie. Ein weiteres Beispiel ist das Wort „Zelle“, das, je nach Kontext, einen Bezug zur Informationstechnologie, zum Terrorismus oder zum Strafvollzug aufweisen kann, um nur die naheliegenden Kontexte zu nennen. ${ }^{25}$ Doch gerade der Kontext, aus dem sich die Bedeutung mancher Wörter erst ergibt, bleibt bei einer einfachen Stichwortsuche unberücksichtigt. Historikerinnen und Historiker dürften jedoch meist an einem spezifischen Kontext

\footnotetext{
${ }^{22}$ Eine Alternative bestünde darin, nicht die Zahl der Wortvorkommen, sondern die derjenigen Artikel zu erheben, in denen das Wort auftritt, und diese wiederum in Verhältnis zur Anzahl aller Artikel zu setzen.

${ }^{23}$ Diese gelten natürlich auch für Tools wie den Google Ngram Viewer.

${ }^{24}$ Martin L. Müller, „Turnen und Sport im sozialen Wandel: Körperkultur in Frankfurt am Main während des Kaiserreichs und der Weimarer Republik“, Archiv für Sozialgeschichte, 33 (1993), S. 107-136.

${ }^{25}$ Ein ähnlich gelagertes Beispiel führen wir mit dem Wort [Index] in unserem Ursprungsbeitrag an. Siehe Buchner, Jopp, Spoerer und Wehrheim, „Zur Konjunktur des Zählens - oder wie man Quantifizierung quantifiziert. Eine empirische Analyse der Anwendung quantitativer Methoden in der deutschen Geschichtswissenschaft“, S. 606. Gerade für Tools, die keinen Einblick in die konkreten Suchtreffer erlauben, sind false positives ein nicht zu unterschätzendes Problem, da man deren Häufigkeit im Grunde nicht abschätzen kann.
} 
bzw. einer spezifischen Bedeutungsausprägung interessiert sein. Entsprechend muss sichergestellt werden, dass auch wirklich nur die gesuchte Bedeutungsvariante des Suchbegriffs erfasst wird. In diesem Zusammenhang ergibt sich ferner das Problem, dass eine einfache Stichwortsuche keine lexikalischen Veränderungen eines Begriffs im Zeitablauf berücksichtigt, Bedeutungsänderungen eines Wortes werden also ausgeblendet. So kann ein Wort beispielsweise über einen Zeitraum zwar konstant auftreten, dabei aber seine Bedeutung verändern. Zudem gilt es, eventuell verschiedene historische Schreibweisen zu berücksichtigen, was insbesondere die Auswertung mittelalterlicher und frühneuzeitlicher Quellen erschwert. Auch wenn für die genannten Fallstricke verschiedene technische Lösungen zur Verfügung stehen, so zeigen sie doch deutlich, welche Aspekte bereits bei einer einfachen Stichwortsuche berücksichtigt werden müssen.

Suchbegriffe. Viertens lässt sich die konkrete Suchanfrage nach einem Begriff auf verschiedene Weise formulieren. So kann entweder nach einem einzelnen feststehenden Ausdruck wie [turn] oder nach Flexionen beziehungsweise Wortstämmen und Trunkierungen gesucht werden, was zum Teil sehr unterschiedliche Trefferzahlen liefert. ${ }^{26}$ Je nach Suchbegriff erscheinen unterschiedliche Vorgehensweisen zielführend, die sich vor allem über Platzhalter (*) und die Großund Kleinschreibung realisieren lassen. Natürlich ließe sich auch nach mehreren Begriffen bzw. Mehrworteinheiten (Bigrams wie cultural turn, Trigrams, etc.) suchen, worauf wir jedoch in Anbetracht der hohen Zahl vorgeschlagener Unigramme, also Einworteinheiten, verzichten. ${ }^{27}$ Hier stellt sich die ganz grundsätzliche Frage, woran man als Suchender eigentlich interessiert ist. Geht es beispielsweise um die allgemeine Verbreitung des Begriffs „Diskurs“? In diesem Fall böte sich vermutlich eine Wortstammsuche nach diskurs* an, um beispielsweise auch Komposita wie „Herrschaftsdiskurs“ oder Adjektive wie „diskurstheoretisch“ zu erfassen. Ist man dagegen am konkreten Einsatz der Methode „Diskursanalyse“ interessiert, wäre wohl ein engerer Suchbegriff wie *diskursanaly* sinnvoll. Die Frage nach dem ,richtigen“ Suchbegriff stellt sich insbesondere dann, wenn nach Personen gesucht werden soll. Nutzt man Vor- und Nachnamen, so ist zwar sichergestellt, dass die gesuchte Person auch höchstwahrscheinlich gefunden wird. Aber das gilt zum einen nicht für geläufige Kombinationen wie „Peter Müller“28

\footnotetext{
${ }^{26}$ Die Wortstammsuche gestaltet sich insofern problematisch, als manche Ausdrücke auch in Begriffen enthalten sich, die mit dem Suchwort nichts zu tun haben. So steckt der Ausdruck „,gender“ in seiner englischen, kleingeschriebenen Variante beispielsweise im Wort „folgendermaßen“.

${ }^{27}$ Hier ist auch auf die Möglichkeit der Nutzung Boolescher Suchoperatoren zu verweisen, die in vielen Suchmasken Verwendung finden.

${ }^{28}$ Wie der Spiegel kürzlich feststellte, heißen Professoren in Deutschland gegenwärtig besonders häufig Hans, Klaus, Peter oder Wolfgang. Vgl. „Professoren heißen Hans, Klaus oder Peter“, in: Spiegel online vom 13.02.2019, verfügbar unter https://www.spiegel.de/lebenundlernen/uni/professoren-vornamen-hans-klaus-peter-sind-amhaeufigsten-a-1253011.html (Zugriff am 01.10.2020).
} 
und entspricht zum anderen nicht ganz dem Schreibgebrauch, da hier meist nur der Nachname verwendet wird. Sucht man dagegen nur den Nachnamen, ist Verwechslungsgefahr selbst bei weniger geläufigen Namen wie „Koselleck“ (Arno oder Reinhart?) gegeben. ${ }^{29}$ Mischt man beide Ansätze, sind die Zahlen in quantitativer Hinsicht nicht vergleichbar. Letztlich haben wir uns für die Suche nach Vor- und Nachnamen entschieden, um das Risiko falsch-positiver Treffer zu minimieren, wodurch die Trefferzahl jedoch vergleichsweise niedrig ausfällt. ${ }^{30}$ Grundsätzlich stellt sich die Frage nach den Suchbegriffen bereits auf der konzeptionellen Ebene. In unserem Fall ergab sich die Auswahl durch eine nicht-repräsentative Umfrage auf Twitter, ergänzt durch unsere persönlichen Kandidaten, was zugegebenermaßen einen eklektischen Charakter hat. Natürlich ließe sich die Auswahl der Begriffe auch auf dieser Ebene systematisieren, indem man entweder von einschlägigen Lexika und Glossaren ausgeht oder die Umfrage in anderer Weise systematisiert. ${ }^{31}$

Kategorisierung. Fünftens ist im Folgenden eine Vielzahl von Begriffen darzustellen, was nach einer Systematisierung der Präsentation verlangt. Entsprechend haben wir die Begriffe in die Kategorien „Inhalte“, „Personen“, „Methoden“ und „Sonstige Begriffe“ eingeteilt, wobei natürlich manche Suchwörter in mehr als einer Kategorie verortet werden können. ${ }^{32}$ Wenn man beispielsweise nach [Sozialgeschichte] sucht, meint man dann einen Gegenstand geschichtswissenschaftlicher Forschung oder einen Forschungsansatz? Aufgrund solcher Ambiguitäten sind die gewählten Kategorien primär als Lesehilfen zu verstehen. Zudem sei erneut darauf hingewiesen, dass aus Gründen der Transparenz konkrete Suchbegriffe mittels der in Fußnote 5 erörterten Notation hervorgehoben sind.

\footnotetext{
${ }^{29}$ Als besonders problematisches Beispiel wäre in unserem Fall auch auf die Historikerfamilie Mommsen zu verweisen.

${ }^{30}$ Konkret wurden jeweils drei Kombinationen gesucht, die an folgendem Beispiel verdeutlicht werden sollen: [Reinhart Koselleck], [Reinhart Kosellecks], [Koselleck, Reinhart]. Letzteres wurde genutzt, um auch Verweise in Literaturangaben zu erfassen. Ein alternatives Vorgehen bestünde beispielsweise darin, mit Hilfe der sogenannten Named Entity Recognition gezielt alle Referenzen auf Personen zu extrahieren. Siehe beispielsweise Kai Labusch, Clemens Neudecker und David Zellhöfer, „BERT for Named Entity Recognition in Contemporary and Historical German", Proceedings of the 15th Conference on Natural Language Processing (KONVENS 2019) (2019), S. na.

${ }^{31}$ Für den lexikalischen Ansatz böte sich vor allem die Arbeit von Reinhart Koselleck an. Siehe etwa Koselleck, Begriffsgeschichten; Brunner, Conze und Koselleck, Hrsg., Geschichtliche Grundbegriffe. Historisches Lexikon zur politisch-sozialen Sprache in Deutschland. Damit ließe sich sicherstellen, dass die Auswahl anhand aus Sicht der Zeitgenossen relevanten Kriterien folgt, während der Ansatz der Umfrage zwangsläufig einen Fokus auf die Gegenwart lenkt. Die in unserem Ursprungsaufsatz gesuchten Begriffe stammen beispielsweise aus Glossaren statistischer Lehrbücher.

${ }^{32}$ Wir haben beispielsweise $\{$ Hitler\} und \{Bismarck\} dem Bereich „Inhalte“ zugeordnet, da diese stärker als die übrigen betrachteten Personen einen Untersuchungsgegenstand bildeten.
} 
Inhalte. In der ersten Kategorie „Themen“ ließe sich angesichts der Themenvielfalt in der geschichtswissenschaftlichen Forschung natürlich nach unzähligen Begriffen suchen. Da wir uns hier jedoch qua Korpuskonstruktion auf die deutschsprachige Geschichtswissenschaft beschränken, ist es naheliegend, zunächst die Beschäftigung mit dem eigenen Land abzufragen. Sucht man etwa nach \{Deutschland\} und \{deutsch\}, so ergeben sich 335.732 Treffer, was nicht weniger als 356 Treffern auf 100.000 Wörtern entspricht. Diese verteilen sich auf 8.498 Artikel, was impliziert, dass in gut 94 Prozent aller Aufsätze mindestens einmal der Suchbegriff genannt wird. Im Vergleich dazu erbringt eine Suche nach \{Frankreich\} und \{französisch\} leidglich 79.486 Treffer in 6.243 Artikeln. Betrachtet man den Verlauf beider Reihen (siehe Abbildung 3), so ergibt sich ein starker Anstieg für den Bezug auf Deutschland bis etwa 1970, gefolgt von einem stabilen Niveau, bis es 2010 zu einem Abfall kommt. Dagegen entwickelt sich die „Franzosen-Kurve“ auf ihrem wesentlich niedrigeren Niveau relativ stabil. Wenn wir uns verschiedenen Perioden der deutschen Geschichte zuwenden, zeigt sich für den Ausdruck *nationalsozial* ein bis Ende der 1990er Jahre sehr volatiler, aber steigender Trend, während sich für \{Hitler\} bis Ende der 1970er Jahre besonders hohe Werte finden. Der Ausdruck *holocaust* bewegt sich im Vergleich zu diesen beiden Ausdrücken auf sehr niedrigem Niveau und taucht überhaupt erst im Jahr 1978 in einem Aufsatz von Rolf Steininger auf, allerdings in einem englischsprachigen Zitat. ${ }^{33}$ Eine erste größere Zahl Suchtreffer (55 in sechs Aufsätzen) ergibt sich für das Jahr 1979, die jedoch zum Hauptteil auf die beiden Artikel von Martin Broszat und Wolfgang Scheffler zur Fernsehserie „Holocaust“ entfallen. ${ }^{34}$ Aber auch danach konzentrieren sich die Suchtreffer lediglich auf einzelne Jahre. ${ }^{35}$ Der Begriff [Shoa] ist, auch wenn man verschiedene Schreibweisen berücksichtigt, sogar nur 41 mal zu finden. Weitere Zeitabschnitte der deutschen Geschichte lassen sich mit \{Deutsches Kaiserreich\} und [Weimarer Republik] verschlagworten, wobei für ersteres interessanterweise nur recht wenige Treffer (1.303) auftreten, die einen bis 1990 ansteigenden, danach bis 2005 fallenden und im Anschluss erneut steigenden

\footnotetext{
${ }^{33}$ Rolf Steininger, „England und die deutsche Gewerkschaftsbewegung 1945/46“, Archiv für Sozialgeschichte, 18 (1978), S. 79.

${ }^{34}$ Martin Broszat, „,Holocaust“ und die Geschichtswissenschaft“, Vierteljahrshefte für Zeitgeschichte, 27 (1979), S. 285-298; Wolfgang Scheffler, „Anmerkungen zum Fernsehfilm ,Holocaust“ und zu Fragen zeithistorischer Forschung“, Geschichte und Gesellschaft, 5 (1979), S. 570-579. Siehe auch Frank Bösch, „Versagen der Zeitgeschichtsforschung?", Zeithistorische Forschungen, 6 (2009), S. 477-482.

${ }^{35}$ Die drei Aufsätze mit den meisten Suchtreffern sind Frank Bajohr, „Zwei Jahre Zentrum für Holocaust-Studien am Institut für Zeitgeschichte“, Vierteljahrshefte für Zeitgeschichte, 64 (2016), S. 139-149; Jacob S. Eder, „Ein »Holocaustsyndrom«? Die politischen Beziehungen zwischen der Bundesrepublik und amerikanischjüdischen Organisationen in den 1980er Jahren“, Archiv für Sozialgeschichte, 52 (2012), S. 633-665; Michael Schmitz, „Ein deutsches Denkmal. Das Holocaust-Denkmal im Brennpunkt deutscher Erinnerungspolitik“, Archiv für Kulturgeschichte, 87 (2005), S. 165-193.
} 
Verlauf aufweisen. Demgegenüber weist die „Bismarck-Kurve“ (13.848 Treffer) einen insgesamt fallenden Trend auf. [Weimarer Republik] (8.889 Treffer) zeigt wie \{Deutsches Kaiserreich\} einen zunächst steigenden Verlauf, der sich ab Mitte der 1980er Jahre jedoch umdreht. Für [Bundesrepublik] und [BRD], die zusammen auf 20.379 Treffer kommen, zeichnet sich ab den 1970er Jahren ein steiler Anstieg ab, der seinen Höhepunkt in den Jahren 2008 bis 2013 findet, was zeitlich mit dem Boom der zeithistorischen, von Anselm Doering-Manteuffel und Lutz Raphael initiierten „Nach dem Boom“-Literatur zusammenfällt. ${ }^{36}$ Zugleich ist in diesen Jahren ein starker Ausschlag für den kombinierten Ausdruck [DDR] und \{Deutsche Demokratische Republik\} (20.430 Treffer) ${ }^{37}$ zu beobachten, ebenso wie für das Jahr 1999, was neben dem zehnten Jahrestag des Mauerfalls in erster Linie auf ein Schwerpunktheft des Archivs für Sozialgeschichte zur Sozialgeschichte der DDR zurückzuführen ist. ${ }^{38}$ Ein letzter in diesen Kontext zu verortender Suchbegriff ist *sonderweg*, dessen Verlauf auf den ersten Blick darauf hindeutet, dass die Frage nach einer deutschen Sonderrolle zwischen 1980 und 2000 zunehmend thematisiert wurde, wobei ein erneuter Anstieg gegen Ende des Betrachtungszeitraums auffällt. Allerdings wird etwa anhand der Aufsätze von Mathias Häusler und Bernd Weisbrod deutlich, dass der Begriff des Sonderwegs nicht zwangsläufig für den deutschen Fall reserviert ist. $^{39}$

Welche weiteren themenspezifischen Begriffe bieten sich für eine Suche an? Angesichts der thematischen Vielfalt in der deutschen Geschichtswissenschaft und der Breite des Korpus stellt jede Auswahl zwangsläufig eine Verkürzung dar. Dabei bietet es sich an, sich auf größere Entwicklungsströme zu konzentrieren, womit man im Falle der deutschen Geschichtswissenschaft

\footnotetext{
${ }^{36}$ Anselm Doering-Manteuffel, „Nach dem Boom: Brüche und Kontinuitäten der Industriemoderne seit 1970“, Vierteljahrshefte für Zeitgeschichte, 55 (2007), S. 559-581; Anselm Doering-Manteuffel und Lutz Raphael, Nach dem Boom: Perspektiven auf die Zeitgeschichte seit 1970 (Göttingen, 2012); Wolfgang Schroeder und Samuel Greef, „Gewerkschaften und Arbeitsbeziehungen nach dem Boom“, in Anselm Doering-Manteuffel, Lutz Raphael und Thomas Schlemmer, Hrsg., Vorgeschichte der Gegenwart (Göttingen, 2016), S. 245-270; Morten Reitmayer und Thomas Schlemmer, Hrsg., Die Anfänge der Gegenwart: Umbrüche in Westeuropa nach dem Boom (München, 2014).

${ }^{37}$ Dass Bundesrepublik und DDR auf nahezu identische Verweiszahlen kommen, mag auf den ersten Blick erstaunen. Es sei jedoch darauf hingewiesen, dass beide Suchoperationen sowohl die jeweilige Abkürzung als auch die ausgeschriebene Bezeichnung enthalten, wobei [DDR] wesentlich häufiger auftritt als [BRD] (19.939 gegenüber 1.203 Treffer). Dies dürfte damit zu erklären sein, dass „DDR“ regelmäßig in Komposita wie „DDR-Bürger“ oder „DDR-Vergangenheit“ zu finden ist, wohingegen die politisch zeitweilig sehr aufgeladene Abkürzung „BRD“ wesentlich seltener verwendet wurde. Siehe dazu auch Stefan Schmidt, „Die Diskussion um den Gebrauch der Abkürzung ,BRD““, Fachinformationen und Analysen des wissenschaftlichen Dienstes des Deutschen Bundestags (2009), S. n.a.

38 Siehe Archiv für Sozialgeschichte Band 39 (1999).

${ }^{39}$ Mathias Häußler, „Ein britischer Sonderweg? Ein Forschungsbericht zur Rolle Großbritanniens bei der europäischen Integration seit 1945“, Vierteljahrshefte für Zeitgeschichte, 67 (2019), S. 263-286; Bernd Weisbrod, „Der englische ,Sonderweg“ in der neueren Geschichte“, Geschichte und Gesellschaft, 16 (1990), S. 233-252.
} 
bei der Historischen Sozialwissenschaft und der (Neuen) Kulturgeschichte landet. ${ }^{40}$ Betrachtet man die Entwicklung der beiden Begriffe [Sozialgeschichte] und [Kulturgeschichte], dann wird deutlich, welchen Boom erstere in den 1970er Jahren erfuhr, wobei ein zweiter Anstieg in den 1990er Jahren zu beobachten ist, der mit einem Aufschwung von [Kulturgeschichte] verbunden war. Zu erklären ist dieser zweite Anstieg von [Sozialgeschichte] vermutlich damit, dass in der Debatte um den cultural turn ${ }^{41}$ eben auch eine Diskussion sozialhistorischer Ansätze stattfand. Weitet man die Suchbegriffe auf die Wortstämme *sozial* und *kultur* aus, ergibt sich eine zwar ähnliche Entwicklung, allerdings fällt hier ein enormer quantitativer Unterschied zwischen beiden Begriffen auf. Die wesentlich niedrigeren Trefferzahlen für *kultur* könnten damit zu erklären sein, dass die Hinwendung zu kulturellen, auf individuelle Aspekte abzielenden Fragestellungen im Rahmen des cultural turns ein facettenreicher Vorgang war, der sich in mehreren Teil-turns wie dem linguistic turn, dem spatial turn oder dem iconic turn vollzog ${ }^{42}$ und dementsprechend mit dem einfachen Begriff „Kultur“ nur unvollständig abgebildet wird. Tatsächlich lässt sich in den 1980er Jahren ein Anstieg etwa der Suchbegriffe *alltag*, *mentalität* bzw. \{mental\}, *identität* und *kommunikation* bzw. *kommunikativ* beobachten.

\footnotetext{
${ }^{40}$ Georg G. Iggers, Geschichtswissenschaft im 20. Jahrhundert. Ein krititischer Überblick im internationalen Zusammenhang (Göttingen, 2007). Hier ließe sich natürlich argumentieren, dass die Begriffe Sozial- und Kulturgeschichte auch im Bereich der Methoden betrachtet werden könnten.

${ }^{41}$ Doris Bachmann-Medick, Cultural Turns. Neuorientierungen in den Kulturwissenschaften (Hamburg, 2010); Christoph Conrad, „Die Dynamik der Wenden. Von der neuen Sozialgeschichte zum cultural turn“, Geschichte und Gesellschaft, 22 (2006), S. 133-160; Ute Daniel, „Geschichte schreiben nach der ,kulturalistischen Wende“"“, Archiv für Sozialgeschichte, 43 (2003), S. 576-599; Iggers, Geschichtswissenschaft im 20. Jahrhundert. Ein krititischer Überblick im internationalen Zusammenhang; Thomas Mergel, „Cultural Turns and Political History“, Ricerche di storia politica (2017), S. 33-42; Peter Schöttler, Geschichtswissenschaft vor und nach dem ,linguistic turn “ (Münster, 2018); Peter Schöttler, „Wer hat Angst vor dem ,linguistic turn“?“, Geschichte und Gesellschaft, 23 (1997), S. 143-151.

${ }^{42}$ Bachmann-Medick, Cultural Turns. Neuorientierungen in den Kulturwissenschaften.
} 


\section{Abbildung 3 Begriffe der Kategorien Inhalte und Personen}
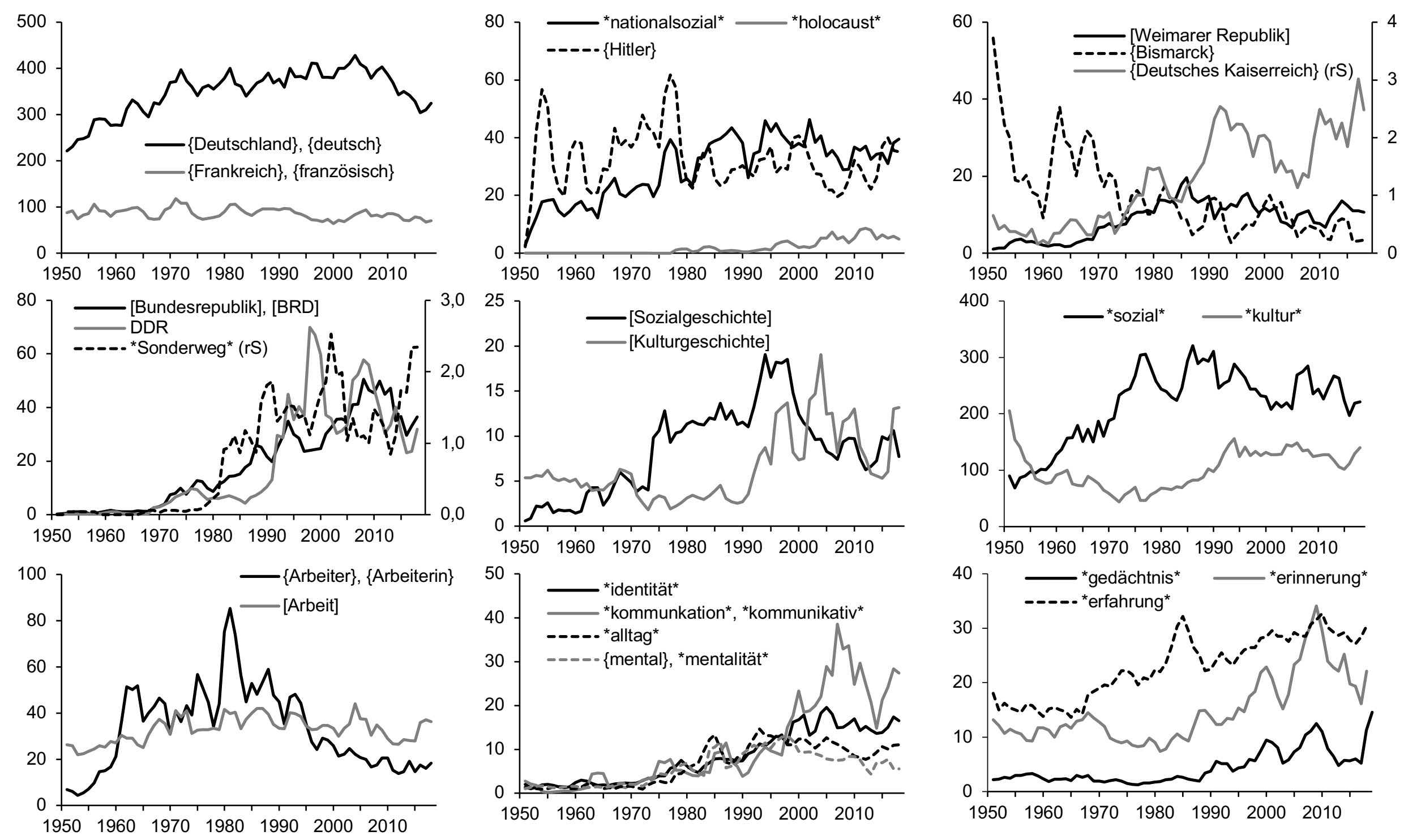

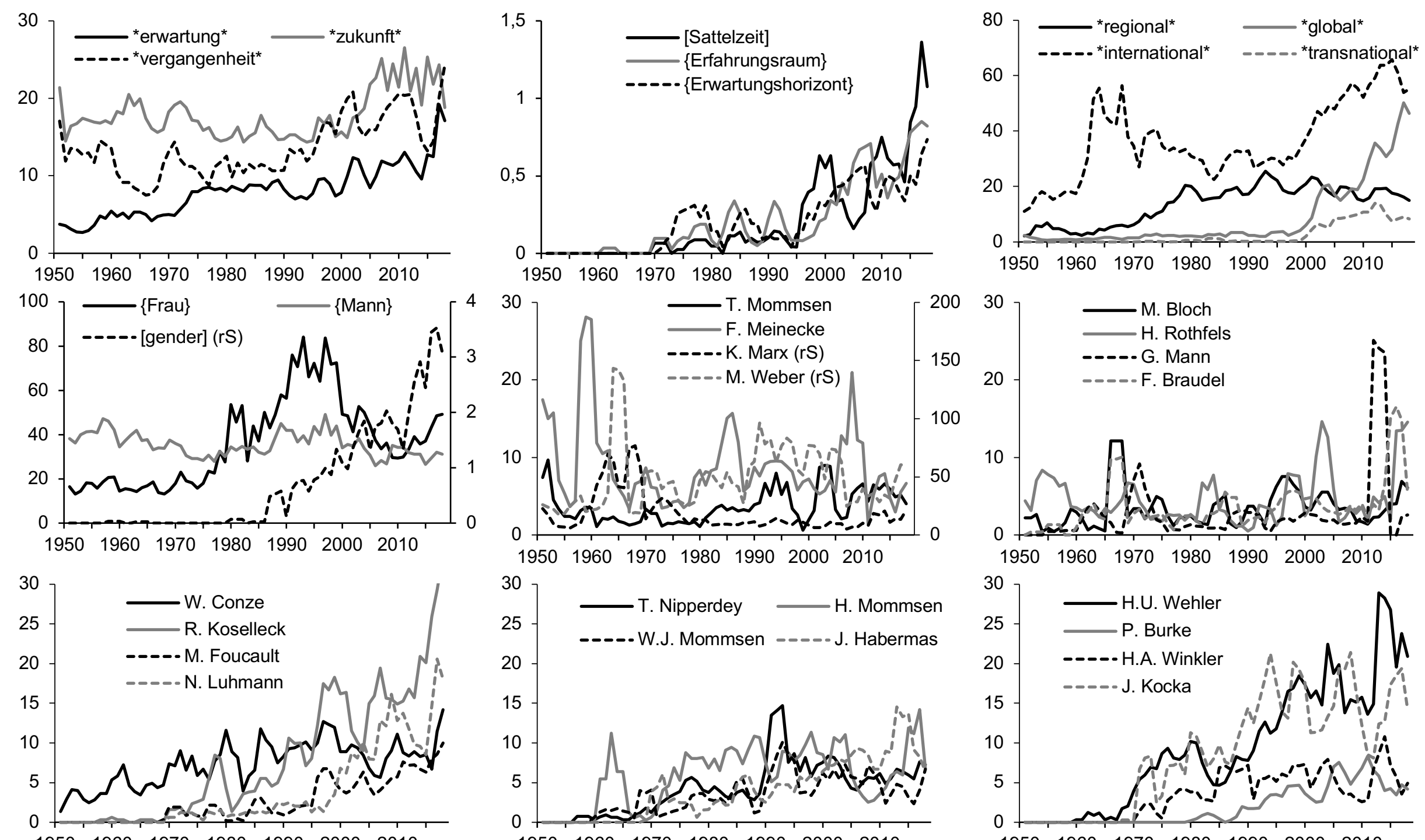

$\begin{array}{lllllll}1950 & 1960 & 1970 & 1980 & 1990 & 2000 & 2010\end{array}$

$\begin{array}{lllllll}1950 & 1960 & 1970 & 1980 & 1990 & 2000 & 2010\end{array}$

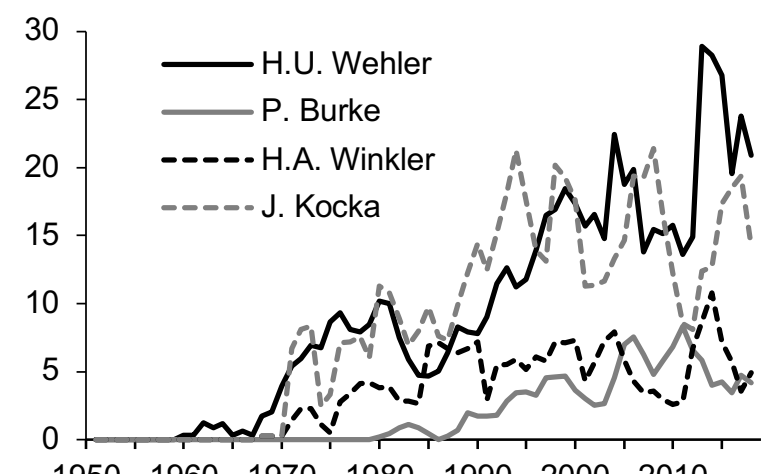

Anmerkungen: Soweit nicht anders angeben, wurden alle Flexionen der Suchbegriffe berücksichtigt. Personen (bis auf Hitler und Bismarck) wurden nach dem Schema „Vorname Nachname“ und „Nachname, Vorname“ gesucht. DDR = \{Deutsche Demokratische Republik\} \& [DDR]. Treffer pro 100.000 Wörter, zentrierte gleitende Drei-Jahresdurchschnitte. Quelle: eigene Abbildungen. 
Weitere Begriffsvorschläge betreffen gewissermaßen die zeitliche Perspektive der Aufsätze. Sowohl *erwartung* als auch *erfahrung* zeigen dabei ansteigende Verläufe, *gedächtnis* findet sich besonders seit 1990. Dagegen bewegt sich *erinnerung* auf einem recht konstanten Niveau. *zukunft* weist hingegen eine ab den 2000er Jahren steigende Häufigkeit auf, während für *vergangenheit* ein solcher bereits seit etwa 1990 zu beobachten ist. In diesem Zusammenhang liegt es natürlich nahe, einige der von Reinhart Koselleck geprägten Begriffe ${ }^{43}$ zu überprüfen. Betrachtet man etwa die drei Begriffe \{Erwartungshorizont $\}$, \{Erfahrungsraum $\}$ und \{Sattelzeit\}, so stellt man fest, dass alle drei ab Mitte der 1990er Jahre an Bedeutung gewinnen.

Neben der zeitlichen lässt sich auch die räumliche Perspektive mit Stichworten erschließen, etwa mit den Begriffen *regional*, *global*, *international* und dem in jüngerer Zeit in Mode gekommenen *transnational*. Alle Kurven weisen einen zum Teil sehr steilen Anstieg seit den frühen 2000er Jahren auf, was auf einen spatial turn schließen lässt. Tatsächlich erlebt die Globalgeschichte momentan einen Boom, wofür der jüngst erschienene, von Andreas Fahrmeir herausgegebene Sammelband „Deutschland. Globalgeschichte einer Nation“44 beispielhaft steht. Allerdings sind die Begriffe *international* und *regional* bereits in den Jahrzehnten davor zu finden, wobei für letzteren ein besonders starker Anstieg in den 1970er Jahren zu beobachten ist. ${ }^{45}$ Ein weiterer, vor allem in jüngerer Vergangenheit populärer Begriff ist [gender]. Hier finden sich zwar insgesamt recht wenige Treffer (799), allerdings wird dieser Begriff seit Mitte der 1980er Jahre zunehmend häufiger verwendet. Sucht man nach den etwas ,altmodischeren“ Begriffen $\{$ Mann $\}$ und $\{F r a u\}$, so ergibt sich ein faszinierender Unterschied. Während \{Mann $\}$ einen leicht negativen Trend aufweist, stellt sich für \{Frau\} ein erheblicher Anstieg zwischen den späten 1970er und späten 1990er Jahren ein, wobei \{Frau\} mit 36.969 Treffern vor \{Mann\} liegt, auf den lediglich 33.012 Treffer entfallen. ${ }^{46}$

\footnotetext{
${ }^{43}$ Reinhart Koselleck, Vergangene Zukunft - Zur Semantik geschichtlicher Zeiten (Frankfurt am Main, 1979); Koselleck, Begriffsgeschichten.

${ }^{44}$ Andreas Fahrmeir, Hrsg., Deutschland. Globalgeschichte einer Nation (München, 2020).

${ }^{45}$ Für *international* besteht jedoch ein erhöhtes Risiko falsch-positiver Suchergebnisse, wie beispielshaft drei Artikel zur „Ersten Internationalen“ zeigen, die im Übrigen auch die Ausschläge der Jahre 1964/67 erklären. Siehe Erich Gruner, „Die Erste Internationale und die Schweiz“, Archiv für Sozialgeschichte, 6/7 (1967), S. 199-239; Miklós Molnár, „Die Londoner Konferenz der Internationale 1871“, Archiv für Sozialgeschichte, 4 (1964), S. 283445; Herbert Steiner, „Die Internationale Arbeiterassociation und die österreichische Arbeiterbewegung“, Archiv für Sozialgeschichte, 4 (1964), S. 447-513.

${ }^{46}$ Beim Suchwort \{Mann\} ergibt sich zudem die Problematik, dass damit erstens der Familienname gemeint sein kann und es zweitens eine Reihe von Nachnamen gibt, die diesen Ausdruck enthalten, wie beispielsweise „Hoffmann". Letzteres wäre bei einer Suche nach $\{$ Mann $\}$ zwar grundsätzlich unproblematisch, leider ergab es sich des Öfteren, dass diese Namen durch einen nicht erkannten Zeilen- oder Seitenumbruch „zerlegt“ wurden. Daher ist der genannte Wert als etwas überhöhte Schätzung aufzufassen.
} 
Personen. Die Suche nach Personen ist sowohl aus technischer Sicht (s.o) als auch gerade inhaltlich betrachtet nicht ganz unproblematisch, da solch eine Zusammenstellung das Ergebnis einer Wertung der Bedeutung der Personen ist. Eine intersubjektiv nachvollziehbare Auswahl könnte bspw. an anerkannten Listen bedeutender Persönlichkeiten ansetzen, ${ }^{47}$ wobei sich auch dann einige Fragen stellen: Soll die Auswahl auf Historikerinnen und Historiker beschränkt werden, oder sollen auch Personen, die für die Entwicklung der Geschichtswissenschaft bedeutsam waren, ohne selbst im engeren Sinne geschichtswissenschaftlich gearbeitet zu haben, in Betracht gezogen werden? Welcher Zeitraum soll betrachtet werden bzw. welche Personen lassen sich sinnvollerweise im gegebenen Zeitraum suchen ${ }^{48}$ Wie rechtfertigt man die Suche nach der einen Historikerin, während man den anderen Historiker ${ }^{49}$ ausschließt? Wie verhält sich die Zahl der Suchtreffer zur Dauer, die eine Person aktiv gewirkt hat? Auch muss hier, noch mehr als zuvor, die Korpusstruktur bedacht werden, da die meisten Personen vermutlich nur in einem gewissen Fachbereich rezipiert werden. Zusammen genommen legen diese Punkte nahe, dass der Einsatz einfacher Suchanfragen für die Personensuche besonders schwierig ist, zumal die Ergebnisse für die einzelnen Individuen im Grunde kaum miteinander zu vergleichen sind. Letztlich haben wir uns dafür entschieden, eine kleine Auswahl an Historikern und solchen Personen zu treffen, die nach allgemeiner Auffassung besonders prägend für die deutsche Geschichtswissenschaft waren, beispielsweise Michel Foucault, und damit weniger auf ,historische Persönlichkeiten“ abzustellen. ${ }^{50}$ Allerdings zeigt sich, dass das gewählte Suchvorgehen dazu führt, dass für die meisten Personen nur sehr wenige Treffer resultieren, was zu sehr volatilen Kurven ohne erkennbaren Trend führte. Daher beschränkten wir uns an dieser Stelle darauf, einige Beispiele anzuführen, für die sich eine substantielle Trefferanzahl mit in einigen Fällen interessanten Verlaufsmustern ergibt. ${ }^{51}$ Namentlich erwähnt seien an dieser Stelle etwa

\footnotetext{
${ }^{47}$ Siehe beispielsweise Lars Hoffmann, Die berühmtesten Historiker (Wiesbaden, 2008); Lutz Raphael, Hrsg., Klassiker der Geschichtswissenschaft (München, 2006). Auch die Wikipedia-Liste von Historikern und Historikerinnen mag an dieser Stelle hilfreich sein. Siehe https://de.wikipedia.org/wiki/Liste von_Historikern des 20. und 21. Jahrhunderts (Zugriff am 14.10.2020).

${ }^{48}$ Hier ergibt sich die Problematik, dass Personen oftmals erst sehr spät oder gar erst posthum rezipiert werden, weshalb in dem hier untersuchten Zeitraum im Grunde nach einem gewissen Zeitpunkt geborene Personen ausscheiden, es sei denn, es handelt sich um absolute „Stars“ der Profession.

${ }^{49}$ Wie u.a. Karen Hagemann zeigen konnte, hat die deutsche Geschichtswissenschaft ein Geschlechterproblem. Vgl. Karen Hagemann, „Gleichberechtigt? Frauen in der bundesdeutschen Geschichtswissenschaft“, Zeithistorische Forschungen, 13 (2016), S. 108-135. Die Unterrepräsentation von Frauen lässt sich auch in dem von uns untersuchten Korpus beobachten: Je nachdem, wie gezählt wird, beträgt der Anteil weiblicher Autoren gerade einmal zwischen 11,3 und 12,5 Prozent. Dass in der folgenden Abbildung keine Frau genannt wird, ist dieser Unterrepräsentation geschuldet.

${ }^{50}$ Ausnahmen sind hier, wie bereits erwähnt, Adolf Hitler und Otto von Bismarck, die im Kontext der Inhalte genannt wurden.

${ }_{51}$ Das bedeutet natürlich nicht, dass es für hier nicht aufgeführte Persönlichkeiten keine solchen Muster gibt, da wir natürlich nur nach einer begrenzten Anzahl an Personen suchen konnten. Zudem könnte man an dieser Stelle diskutieren, inwieweit mittels bereits erwähnter Named Entity Recognition andere, brauchbarere Ergebnisse zu erzielen wären.
} 
Max Weber (4.702), dessen Kurve, abgesehen von einem Ausreißer im Jahr 1965, ${ }^{52}$ vor allem für die Jahre zwischen 1990 und 2005 einen ansteigenden Trend zeigt. Karl Marx kommt, zum Vergleich, dagegen nur auf 1.647 Treffer, deren zeitliche Verteilung sich primär auf die 1960er Jahre konzentriert. Unter den gesuchten Historikern stechen in quantitativer Hinsicht Hans-Ulrich Wehler (1.011), Jürgen Kocka (941), Reinhart Koselleck (893), Werner Conze (750), Friedrich Meinecke (800) und Hans Mommsen (612) hervor, wobei hier der Auswahl der Zeitschriften für die Trefferhäufigkeit eine besondere Rolle zukommt.

Methoden. Es ist zwar zugegebenermaßen schwierig, auf Basis von Stichwortsuchen die Ebenen der Inhalte und diejenige der Methodik zur Untersuchung dieser Inhalte trennscharf abzugrenzen. Lässt man jedoch ein gewisses Maß an Überschneidung zu, so können einige Trends hinsichtlich des geschichtswissenschaftlichen Werkzeugkastens herausgearbeitet werden. Sucht man beispielsweise nach Wörtern, die die Ausdrücke *quanti* und *statisti* enthalten, so ergibt sich in beiden Fällen ein starker Anstieg in den 1970er Jahren, gefolgt von einem mehr oder weniger ausgeprägten Rückgang (siehe Abbildung 4). Ebenfalls in den 1970er Jahren steigt die Kurve für *vergleich* an, wobei sich diese ab Anfang der 1980er auf einem konstanten Niveau stabilisiert. Beides zusammen ließe sich so interpretieren, dass sich der (explizite) historische Vergleich als methodischer Ansatz etablieren konnte, während dies für den Fall quantitativer Verfahren nicht zu beobachten ist. Einen auffallend steilen Anstieg verzeichnen die Kurven für Diskurs und Netzwerk, wobei wir jeweils zwischen einem allgemeinen und einem spezifischen Suchbegriff unterschieden haben. Die Trefferhäufigkeit der allgemeinen Begriffe *diskurs* und *netzwerk* steigt ab den 1980er bzw. ab Mitte der 1990er Jahre dramatisch an, was nahelegt, dass Diskurse und Netzwerke seither eine zunehmende Rolle in der geschichtswissenschaftlichen Forschung spielen. Betrachtet man allerdings die spezifischeren Suchbegriffe *diskursanaly* und *netzwerkanaly*, die auf die konkrete Anwendung von Diskurs- bzw. Netzwerkanalyse abzielen, ergeben sich wesentlich weniger Treffer, was darauf hindeutet, dass beide Methoden erheblich seltener zum Einsatz kommen, als der Gebrauch der allgemeinen Begriffe suggeriert. Im Falle der Suchabfrage *netzwerkanaly* entfallen zudem 64 der gefundenen 168 auf einen einzigen Artikel. ${ }^{53}$ Ähnliches gilt für die Suchbegriffe *system* und *systemtheor*, wobei letzterer einen der wenigen Begriffe mit einem Comeback

\footnotetext{
${ }^{52}$ Dieser ist auf drei Aufsätze aus der Historischen Zeitschrift zurückzuführen, mit denen die $H Z$, ,wenn auch verspätet, des 100. Geburtstags des großen Gelehrten gedenken“ wollte. Alfred Heuss, „Max Webers Bedeutung für die Geschichte des Griechisch-Römischen Altertums“, Historische Zeitschrift, 201 (1965), S. 529.

${ }^{53}$ Dabei handelt es sich um Eva Jullien, „Netzwerkanalyse in der Mediävistik. Probleme und Perspektiven im Umgang mit mittelalterlichen Quellen“, Vierteljahrschrift für Sozial- und Wirtschaftsgeschichte, 100 (2013), S. $135-153$.
} 
darstellt. Sucht man nach dem Ausdruck *theor*, so ergibt sich ein insgesamt steigender Trend, wobei die 1970er und frühen 1980er Jahre offenbar besonders theorielastig waren. *interdisziplin* weist ebenfalls eine seit den 1970er Jahren steigende Frequenz auf. Was den Einsatz digitaler Methoden angeht, so ist der Ausdruck *digital* natürlich ein besonders problematischer Suchbegriff, schließt er doch die Digitalisierung als zu historisierendes Phänomen mit ein. ${ }^{54}$ Wie dem auch sei, mit 803 Treffern taucht der Ausdruck ohnehin nur recht selten auf und lässt sich im Grunde erst seit Mitte der 2000er Jahre regelmäßig beobachten. Der Suchausdruck *begriffsgeschicht* lässt darauf schließen, dass begriffsgeschichtliche Aufsätze in unserem Korpus so gut wie gar nicht zu finden sind. Dagegen weisen die Suchbegriffe [Oral history], *interview*, *zeitzeuge* und *zeitzeugin*, die wir in der Grafik unter „Oral history“ additiv zusammengefasst haben, darauf hin, dass dieser Ansatz seit den frühen 1980er Jahren immer beliebter wird.

Generell besteht ein Problem darin, dass die vorgenommene Analyse zum einen Methoden nur dann identifizieren kann, wenn sie explizit genannt sind. Viele Arbeiten werden in den früheren untersuchten Jahrzehnten vergleichend durchgeführt worden sein, ohne dass man den Vergleich als Methode explizit herausstellte. Zum anderen lässt sich vielfach feststellen, dass Theorieeinsatz in der Einleitung behauptet wird, um dem Anspruch theoriegeleiteten Forschens zu entsprechen. Clifford Geertz“ „dichte Beschreibung“ etwa ist schon oft bemüht worden, um ein empiriegesättigtes Narrativ theoretisch zu überhöhen.

Sonstige. Bei den verbleibenden Begriffen handelt es sich um solche, die sich nicht oder zumindest nicht eindeutig einer der drei zuvor betrachteten Kategorien zuordnen lassen. Dabei geht es unter anderem um Begriffe, die sich als „Modewörter“ im engeren Sinne betrachten lassen, wie beispielsweise *desiderat* oder *spannungsfeld*, wobei beide Begriffe im Zeitablauf einen ansteigenden Trend aufweisen. Gleiches gilt, wenn auch erheblich potenziert, für den Ausdruck *paradigm*, der darauf hindeutet, dass Paradigmen, in welcher Form auch immer, seit den 1970er Jahren offenbar einen Boom erleben. Besonders interessant erscheint uns hier aus wirtschaftshistorischer Perspektive auch der Verlauf des in den 1980er Jahren unter anderem von Paul A. David geprägten Begriffs der „Pfadabhängigkeit“, dargestellt über den Suchausdruck *pfadabhängig*:55 Zum ersten Mal taucht dieser Ausdruck im Jahr 1996 in zwei

\footnotetext{
${ }^{54}$ Diese Zweiteilung lässt sich durch die beiden Aufsätze mit den meisten Treffern (42 bzw. 44) illustrieren: David Gugerli und Hannes Mangold, „Betriebssysteme und Computerfahndung“, Geschichte und Gesellschaft, 42 (2016), S. 144-174; Schwandt, „Digitale Methoden für die Historische Semantik. Auf den Spuren von Begriffen in digitalen Korpora“.

${ }^{55}$ Paul A. David, „Clio and the Economics of QWERTY“, The American Economic Review, 75 (1985), S. 332 337.
} 
Aufsätzen aus Geschichte und Gesellschaft auf, ${ }^{56}$ um sich im Anschluss langsam aber stetig wenn auch auf einem im Vergleich zu anderen Begriffen niedrigeren Niveau - zu etablieren.

\section{Abbildung 4 Begriffe der Kategorie Methode und Sonstige}
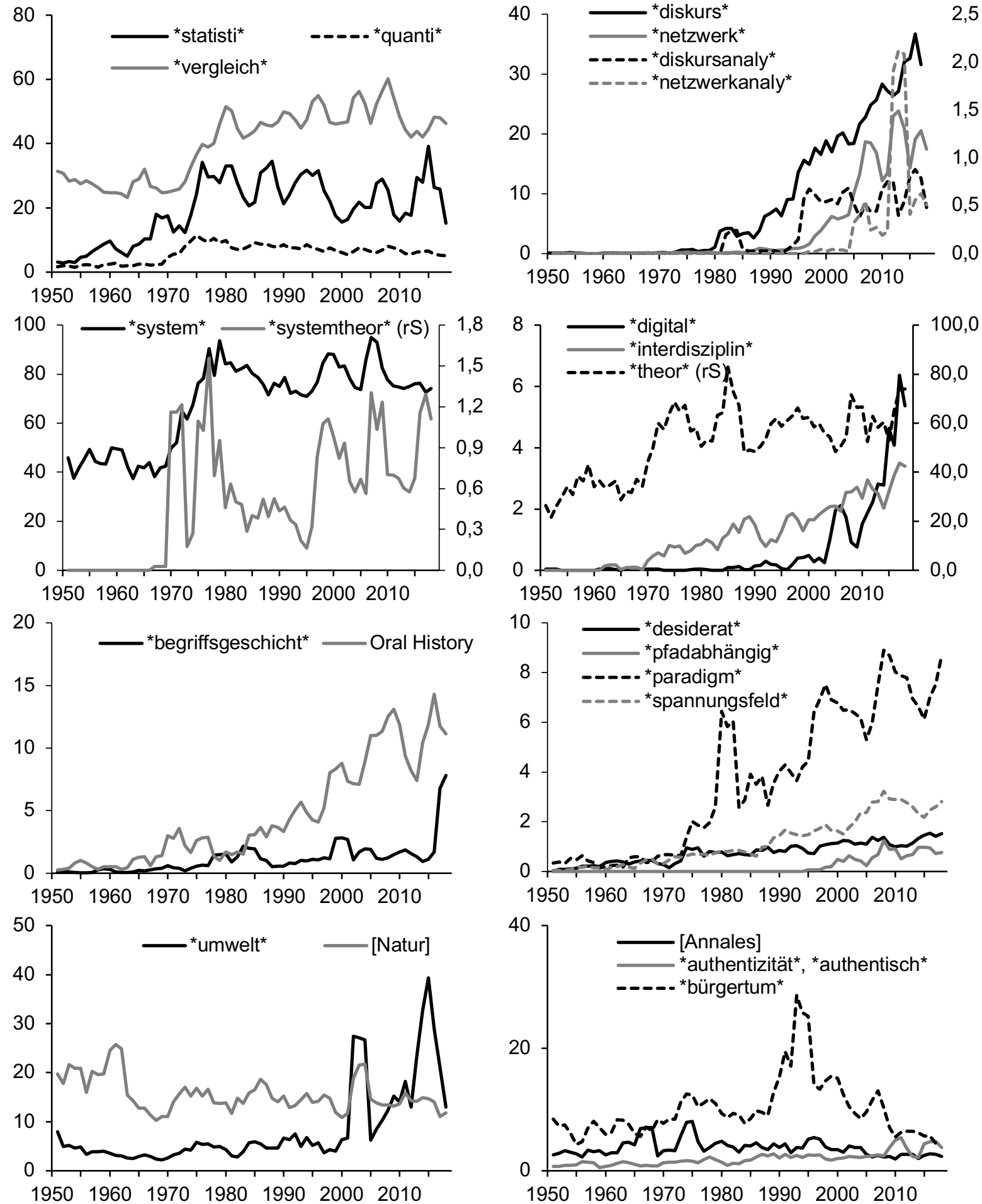

${ }^{56}$ Werner Abelshauser, „Erhard oder Bismarck?“, Geschichte und Gesellschaft, 22 (1996), S. 376-392; Gerhard A. Ritter, „Probleme und Tendenzen des Sozialstaates in den 1990er Jahren“, Geschichte und Gesellschaft, 22 (1996), S. 393-408. 

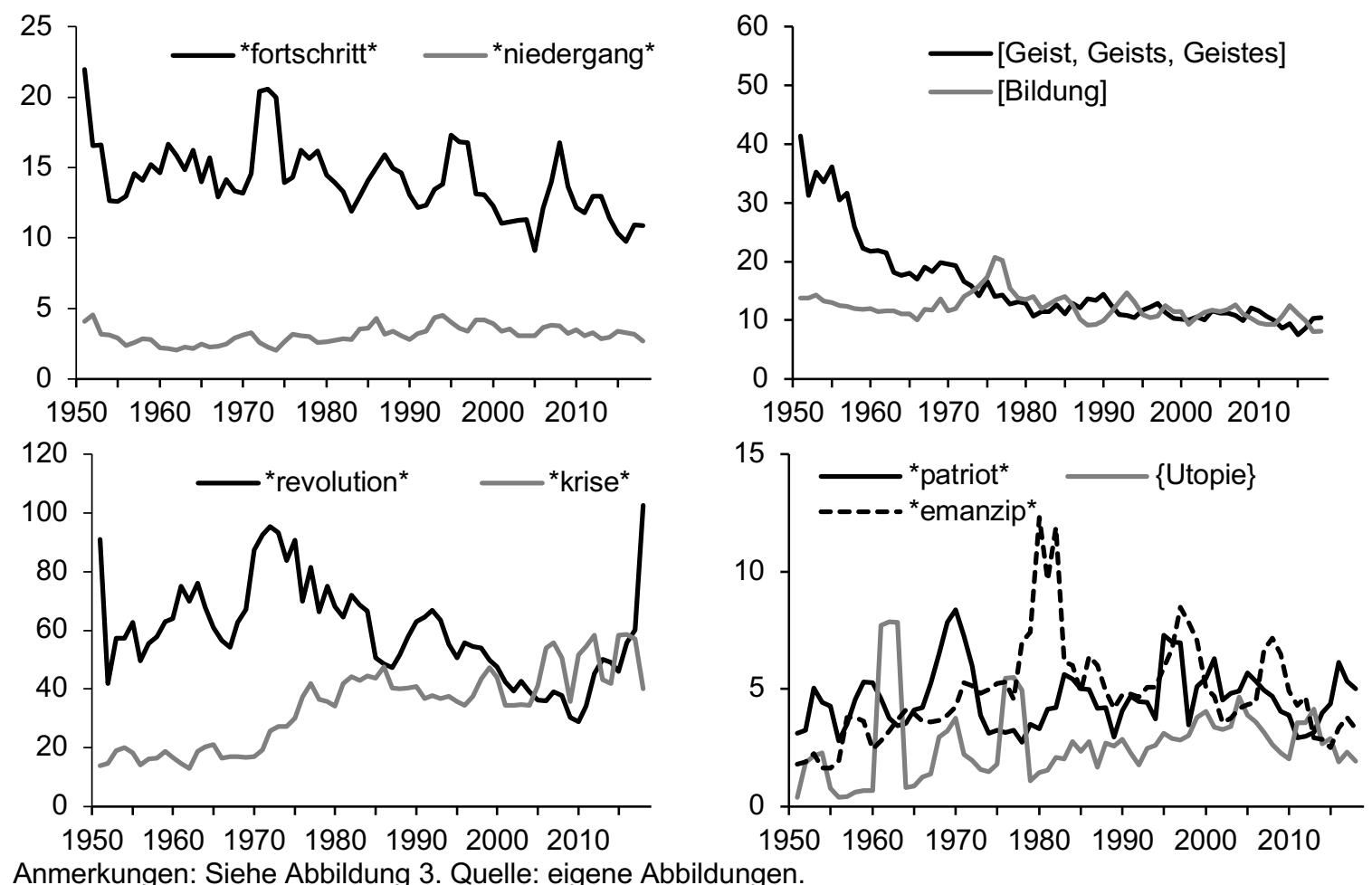

Anmerkungen: Siehe Abbildung 3. Quelle: eigene Abbildungen.

Kommen wir noch einmal auf Reinhart Koselleck zurück. In seinen „Begriffsgeschichten“ finden sich einige Kandidaten bzw. Begriffspaare, die wir abschließend erwähnen möchten. ${ }^{57}$ Dies betrifft zunächst das Begriffspaar *fortschritt* und *niedergang*, deren Verlaufskurven zeigen, dass ersterer augenscheinlich wesentlich häufiger thematisiert wurde als letzterer. Allerdings fällt der Trend für *fortschritt* langfristig, während *niedergang* ein konstantes Thema bildet. Einen ebenfalls fallenden Trend finden wir für die Paarung [Geist, Geists, Geistes] und [Bildung], wobei hier der drastische Rückgang des Geistigen in der ersten Hälfte des Untersuchungszeitraums auffällt. Von den von Koselleck behandelten Einzelbegriffen weisen *patriot*, \{Utopie\} und *emanzip* nur sehr wenige Treffer auf, die sich auf einige wenige Artikel konzentrieren, was sich in sehr volatilen Kurven niederschlägt. Anders sieht es hingegen für *revolution* und *krise* aus. Erstere erlebte einen Höhepunkt um die 1970er Jahre, der hauptsächlich auf Aufsätze aus dem Archiv für Sozialgeschichte zurückzuführen ist. ${ }^{58}$ Zudem fallen die sehr hohen Werte zu Beginn und am Endes des Untersuchungszeitraums ins Auge. ${ }^{59}$ Parallel mit dem Rückgang von *revolution* ist ein Anstieg des Ausdrucks *krise* zu beobachten, der

\footnotetext{
${ }^{57}$ Koselleck, Begriffsgeschichten. Wie erwähnt, bieten Kosellecks andere Arbeiten ebenfalls eine Fundgrube an Suchbegriffen.

${ }^{58}$ Siehe etwa Walter Grab, „Eroberung oder Befreiung? Deutsche Jakobiner und die Franzosenherrschaft im Rheinland 1792 bis 1799“, Archiv für Sozialgeschichte, 10 (1970), S. 7-94; Hartmut Soell, „Weltmarkt Revolution - Staatenwelt. Zum Problem einer Theorie internationaler Beziehungen bei Marx und Engels“, Archiv für Sozialgeschichte, 12 (1972), S. 109-184.

${ }^{59}$ Hier ist vor allem auf den Aufsatz von Theodor Schieder, 'Das Problem der Revolution im 19. Jahrhundert', Historische Zeitschrift, 170 (1950), S. 233-271 sowie das Themenheft des Archivs für Sozialgeschichte von 2019 „Die Welt verändern. Revolutionen in der Geschichte“ zu verweisen.
} 
sich seither auf einem konstanten, ab Mitte der 2000er Jahre nochmals leicht angestiegenem Niveau hält. Ein Beispiel für eine aus dem statistischen Spielen mit Wortfrequenzen resultierenden Forschungsfrage könnte in diesem Kontext darin bestehen, zu untersuchen, inwieweit die offensichtlich zunehmende Thematisierung von vergangenen Krisen in der Literatur der 1970er Jahre mit der zeithistorischen Beschreibung dieser Zeit selbst als genereller Umbruchsund Krisenphase ${ }^{60}$ zusammenhängt.

IV.

Der letzte Punkt leitet zu der Frage über, was wir den Leserinnen und Lesern dieses Textes mit auf den Weg geben möchten. Es ging uns nicht darum, hier tiefere Einblicke in die Geschichte der präsentierten Begriffe oder gar der Geschichtswissenschaft zu liefern, sondern vielmehr darum, eine Diskussion darüber anzuregen, wie mit Hilfe von Begriffsfrequenzen die Entwicklung des Fachs untersucht werden kann und welche methodischen Fallstricke mit einfachen Stichwortsuchen verbunden sind. Dabei sollen unsere Gedanken bewusst als Aufschlag für weitere, mit digitalen Methoden operierende Arbeiten verstanden werden, für die wir abschließend einige weitergehende Überlegungen und Ideen formulieren möchten. ${ }^{61}$

In erster Linie bedarf eine „digitale Begriffsgeschichte“62, oder besser (Fach-) Begriffsgeschichte, wie wir sie hier nur in Ansätzen skizziert haben, einer systematischeren Grundlage. Wie wir bereits mehrfach angemerkt haben, bieten die „Klassiker“ der Begriffsgeschichte einen naheliegenden Ausgangspunkt, wobei unsere Twitter-Umfrage auch an andere Möglichkeiten, wie etwa eine großangelegte Umfrage unter allen Berufshistorikerinnen und -historikern und/oder Geschichtsstudentinnen und -studenten, denken lässt. Gerade dieser eher interaktive $\mathrm{Zu}$ gang gäbe Auskunft darüber, inwieweit sich die Selbstwahrnehmung des Fachs mit der tatsächlichen Forschungspraxis deckt. Daneben wäre darüber nachzudenken, das Korpus zu erweitern und beispielsweise um geschichtswissenschaftliche Reihen zu ergänzen, um damit auch die in der Geschichtswissenschaft noch immer bedeutsame Monographie und den Sammelband als Publikationsformen zu berücksichtigen. Gerade im Hinblick darauf, dass besonders der Forschungsoutput des wissenschaftlichen Nachwuchses in der Qualifikationsphase primär in

\footnotetext{
${ }^{60}$ Doering-Manteuffel und Raphael, Nach dem Boom: Perspektiven auf die Zeitgeschichte seit 1970.

${ }^{61}$ Hier ist in methodischer Hinsicht erneut auf den Aufsatz von Silke Schwandt zu verweisen. Siehe Schwandt, „Digitale Methoden für die Historische Semantik. Auf den Spuren von Begriffen in digitalen Korpora“. Allerdings wird hieran auch deutlich, dass man, sollen mehrere Begriffe analysiert werden, schnell an die Grenzen dessen stößt, was in einem Aufsatz platztechnisch möglich ist.

${ }^{62}$ Friedrich und Biemann, „Digitale Begriffsgeschichte? Methodologische Überlegungen und exemplarische Versuche am Beispiel moderner Netzsemantik“.
} 
monographischer Form erscheint, wäre eine Berücksichtigung von Büchern wünschenswert. Zweitens müssten auf analytischer Ebene die Korrelationen, Interdependenzen und Kookkurenzen der Begriffe berücksichtigt werden, was wir hier aus Platzgründen allerhöchstens andeuten konnten. Es gilt eben, nicht nur die quantitative Entwicklung eines Begriffs innerhalb eines Korpus, sondern auch dessen Bedeutungs- und Kontextveränderungen zu berücksichtigen. In dieser Hinsicht ließe sich überprüfen, wie sich die Entwicklungen bestimmter Begriffe zueinander verhalten, was vor allem für verschiedene Komposita und Mehrworteinheiten eines Wortes wie etwa „Revolution“ (französische vs. industrielle Revolution) interessant wäre. ${ }^{63}$ Drittens wäre stärker zu untersuchen, wie sich die ermittelten Frequenzen auf die Aufsätze verteilen. Wie anhand der genannten Beispiele klar geworden sein sollte, sind manche Begriffe trotz hoher Trefferzahlen nur in sehr wenigen Aufsätzen zu finden, während andere hingegen augenscheinlich einen festen Platz im Sprachrepertoire von Historikerinnen und Historikern einnehmen. Anders formuliert müsste die Konzentration bzw. die Streuung der Begriffe berücksichtigt werden. Viertens bestünde ein weiterer möglicher Ansatz darin, das methodische Vorgehen sozusagen auf den Kopf zu stellen, in dem man nicht von vorher festgelegten Suchbegriffen, sondern vielmehr von den begrifflichen Eigenschaften der Texte selbst ausgeht, also die Analyse quasi agnostisch von empirischen Wortverteilungen aus beginnt. Hierfür bieten sich die zahlreichen digitalen Methoden zur Textauswertung an, die momentan im Bereich der Digital Humanities zunehmend angewendet werden. Neben Ansätzen wie Named Entity Recognition und Word Embeddings ist hier vor allem an das bereits erwähnte Topic Modelling zu denken, mit deren Hilfe die Inhalte großer Textmengen explorativ erschlossen werden können. Zusammenfassend lässt sich der hier präsentierte Aufschlag zu einer digitalen Begriffs- und Historiographiegeschichte also sowohl in der Breite als auch in der Tiefe fortsetzen, und wir hoffen, dass unser Beitrag als weitere Anregung zum Einsatz digitaler Methoden, seien sie einfach oder komplex, dient.

\footnotetext{
${ }^{63}$ Alexander Friedrich und Chris Biemann illustrieren diesen Punkt am Beispiel des „Netzwerks“. Siehe Friedrich und Biemann, „Digitale Begriffsgeschichte? Methodologische Überlegungen und exemplarische Versuche am Beispiel moderner Netzsemantik“.
} 